\title{
Erratum to: Platelet Activation Markers Are Associated with Crohn's Disease Activity in Patients with Low C-Reactive Protein
}

\author{
Hiroshi Takeyama ${ }^{1}$ Tsunekazu Mizushima ${ }^{1} \cdot$ Hideki Iijima $^{2} \cdot$ Shinichiro Shinzaki $^{2}$. \\ Mamoru Uemura $^{1}$ - Junichi Nishimura ${ }^{1}$ - Taishi Hata ${ }^{1}$ Ichiro Takemasa ${ }^{1}$. \\ Hirofumi Yamamoto ${ }^{1}$ Yuichiro Doki ${ }^{1} \cdot$ Masaki Mori $^{1}$
}

Published online: 12 August 2015

(C) Springer Science+Business Media New York 2015

\section{Erratum to: Dig Dis Sci}

DOI 10.1007/s10620-015-3745-2

The original version of this article unfortunately contained an error in the coauthor name. The name should be Shinichiro Shinzaki, but was submitted and published as Shinzaki Shinichiro.

The online version of the original article can be found under doi:10.1007/s10620-015-3745-2.

Tsunekazu Mizushima

tmizushima@gesurg.med.osaka-u.ac.jp

1 Department of Surgery, Gastroenterological Surgery,

Graduate School of Medicine, Osaka University, 2-2

Yamada-oka, Suita City, Osaka 565-0871, Japan

2 Department of Gastroenterology and Hepatology, Graduate School of Medicine, Osaka University, 2-2 Yamada-oka, Suita City, Osaka 565-0871, Japan 